\title{
Zurück auf den Rasen
}

\section{BEHANDLUNG EINES PROFIFUSSBALLERS MIT HAMSTRINGVERLETZUNG Es gibt viele unterschiedli-} che Meinungen darüber, wie akute Hamstring-Verletzungen rehabilitiert werden sollten. Oft verlassen sich Sportphysios bei der Gestaltung des Rehaprogramms für einen verletzten Athleten vor allem auf ihre persönlichen Erfahrungen und Präferenzen. Der Rehaverlauf ist häufig rein zeitgesteuert oder basiert auf willkürlich gewählten Tests, die nicht auf die spezifischen Erfordernisse der Sportart abgestimmt sind. Wie die Rehabilitation eines Athleten mit Hamstringverletzung anhand der aktuell verfügbaren Evidenz aussehen sollte, zeigt dieser Fallbericht. Nicol van Dyk, Arnlaug Wangensteen und Rod Whiteley

\section{Einleitung}

Die Rehabilitation einer akuten Hamstring-Verletzung stellt nach wie vor eine Herausforderung dar, und in der Fachliteratur findet der Kliniker eine Vielzahl unterschiedlicher Übungsoptionen und Rehabilitationsprotokolle [12, 14, 20, 21, 22]. Trotz des vielfältigen Angebots an Übungen und anderen Interventionen gibt es nur wenig Evidenz dafür, dass bestimmte Behandlungsansätze anderen überlegen sind, geschweige denn eindeutige Indikationen, zu welchem Zeitpunkt der Rehabilitation bestimmte Übungen eingesetzt werden sollten. Eine Ausnahme sind Lengthening Exercises (exzentrische Übungen). In einer aktuellen Metaanalyse konnte anhand von zwei Studien gezeigt werden, dass diese Übungen den Return to Play signifikant beschleunigen können [17]. Eine Studie beschäftigte sich mit Spitzenfußballspielern [4], die andere mit Spitzensprintern und -springern [3].

In diesem Fallbericht über eine typische akute, beim Laufen erlittene Hamstring-Verletzung beschreiben und befürworten wir die Anwendung eines kriterienbasierten Protokolls für den Verlauf der Rehabilitation. Außerdem diskutieren wir klinische Prädiktoren, anhand derer Entscheidungen über den Zeitpunkt des Return to Play getroffen werden, und vereinen 40 Jahre klinische Erfahrung in der Sportphysiotherapie mit wissenschaftlicher Dokumentation von Ergebnismessungen mit mehr als 150 akuten Hamstring-Verletzungen.

\section{Fallpräsentation}

Hassan* ( Pseudonym) ist ein 24-jähriger professioneller Fußballspieler, der während eines Liga-Spiels eine Verletzung des hinteren Oberschenkels erlitten hat. Die Verletzung ereignete sich ohne gegnerische Einwirkung in der 80. Minute bei einem Sprint zum Ball mit einem Richtungswechsel. Er konnte das Spiel nicht fortsetzen und verspürte unmittelbar nach dem Vorfall beim Gehen starke, im posterioren Oberschenkel lokalisierte Schmerzen. Die Erstversorgung bestand darin, dass er, gestützt auf einen Assistenten, das Spielfeld verließ, gefolgt von Eisbehandlung, Kompression und Hochlagerung des verletzten Beins. Das Bein wurde teilimmobilisiert, um eine Schmerzprovokation zu verhindern.

Am nächsten Tag wurde Hassan im Aspetar Orthopaedic and Sports Medicine Hospital untersucht. Anwesend bei der Untersuchung waren der Vereinsarzt und ein Physiotherapeut. Der Sport- arzt führte eine umfassende klinische Erstuntersuchung durch, zusätzlich wurde eine MRI-Untersuchung durchgeführt. Das MRI ergab positive Zeichen für eine Verletzung, die einem Riss zweiten Grades des M. biceps femoris an der proximalen Muskel-Sehnen-Verbindung entsprach. Hassan erzählte, dass es sich um seine erste Hamstring-Verletzung handelte und dass er während der letzten fünf Jahre keine sonstigen schweren Verletzungen erlitten hatte. Es gab keine Hinweise auf eine neurale Beteiligung, und die Erstuntersuchung zeigte auch keine weiteren Verletzungen.

\section{Hintergrund}

Der zentrale Grundsatz des Rehabilitationsprotokolls ist, dass bestimmte, vorab festgelegte Kriterien (spezifische physische Tests) erfüllt sein müssen, bevor die nächste Phase der Rehabilitation eingeleitet wird. Tägliche Messungen der subjektiven Schmerzempfindung, der Schmerzen bei Palpation, des Bewegungsumfangs, der Flexibilität und der Muskelkraft ermöglichen es dem Kliniker, auf Grundlage des aktuellen Zustandes die Behandlung anzupassen und die Reaktion auf die Behandlung des Vortages zu identifizieren. Auch wenn wir spezifische Übungen und Vorgehensweisen für jede Phase der Rehabilitation vorschlagen, ist ein kontinuierliches Clinical Reasoning durch den Kliniker erforderlich, um das Protokoll in jeder Sitzung optimal ausführen zu können. Das Clinical Reasoning beinhaltet die Berücksichtigung von Faktoren wie der vermutete Verletzungsmechanismus (Trat die Verletzung während der Schwung- oder der Standphase auf? Handelt es sich um eine aktive oder passive Dehnungsverletzung?), sportspezifische Belastungen der Hamstring-Muskulatur und individuelle Risikofaktoren wie Rumpfstabilität und Lenden-Becken-Kontrolle [15].

Da Belastungen des verheilenden Gewebes über seine Elastizitätsgrenzen hinaus zu einer weiteren Zustandsverschlimmerung führen können - was daran zu erkennen ist, dass bei den Belastungen Schmerzen auftreten -, empfehlen wir, grundsätzlich alle Übungen im schmerzfreien Bereich auszuführen bzw. diesen Bereich nur minimal zu überschreiten [11]. Wenn eine Übung oder Bewegung Schmerzen im Verletzungsbereich auslösen, muss diese Übung oder Bewegung sofort angepasst oder beendet werden. Da unkontrollierte Bewegungen des Beckens sich bei belastungsintensiven Aktionen (z.B. Sprinten) nachteilig auf die Belastung 
der Hamstrings auswirken können, muss immer darauf geachtet werden, dass der Patient seine Übungen mit adäquater Kontrolle und Stabilisation von Hüfte und Rumpf ausführt [20].

Die Aktivität der medialen und lateralen Hamstrings kann gesteigert werden durch zusätzliche mediale bzw. laterale Tibiarotation. Wenn es der Zustand des Patienten erlaubt, werden diese Bewegungen bei Übungen mit Knieflexionsbewegungen eingesetzt, um den verletzten Muskel zielgerichtet zu therapieren.

Das Rehabilitationsprotokoll umfasst sechs willkürlich festgelegte Phasen: drei „physiotherapeutische“ Phasen und drei sportspezifische Phasen. Überlappungen einzelner Übungen im Übergang von der einen zur nächsten Phase sind zugelassen. Dies garantiert einen optimalen Fluss in der Rehabilitation und die Integration klarer Kriterien für die Progression einzelner Übungen. Der wichtigste Aspekt des Protokolls, der in sämtlichen Phasen wiederholt wird, ist die frühe, aber sichere Wiederaufnahme sportspezifischer Aktivitäten wie repetitives schnelles Laufen und Richtungswechsel (s. Tab. 1).

\section{Das auf Kriterien basierende Protokoll für den Verlauf der Rehabilitation}

Im Folgenden beschreiben wir die sechs Phasen des Rehabilitationsprotokolls, die entsprechenden Kriterien für den Übergang zur nächsten Phase (Abb. 1) sowie die physischen Assessments für das tägliche Monitoring und die Steigerung des Lauftrainings.

\section{Phase 1}

Das Hauptziel dieser Phase ist die Förderung des Heilungsprozesses und die Vermeidung aller provokativen Aktivitäten, die diesen Prozess verlängern könnten. Während der ersten Phase des Heilungsprozesses werden Übungen mit geringer Intensität eingesetzt, um das verheilende Gewebe adäquat zu belasten. Hinzu kommen funktionelle Übungen, die Bewegungsmuster erhalten oder sogar verbessern sollen. Bei normalem Rehabilitationsverlauf kommen in dieser Phase aktive Bewegungen in angenäherter und mittlerer Muskellänge (bezüglich Knie- und Hüftflexion), spezifische Weichteilmobilisationen und isometrische bis leicht konzentrische Übungen zum Einsatz. Zu den spezifischen Übungen gehören beispielsweise isometrische Heel Digs, beidbeiniges Bridging und einbeinige Squats unter Beachtung von Becken- und Beinposition (s. „Spezifische Übungen für Phase 1“).

\section{Kriterien/Physische Tests für den Übergang zu Phase 2}

Phase 2 kann begonnen werden, wenn der Sportler eine schmerzfreie einbeinige Kniebeuge ausführen und auf einem Fahrradergometer fünf Minuten lang einen Power-Output (in Watt) erzeugen kann, der dem doppelten seines Körpergewichts (in kg) entspricht. Diese Kriterien werden in den meisten Fällen schon nach kurzer Zeit erfüllt, und auch Hassan konnte bereits zwei Tage nach der Verletzung mit Phase 2 beginnen.

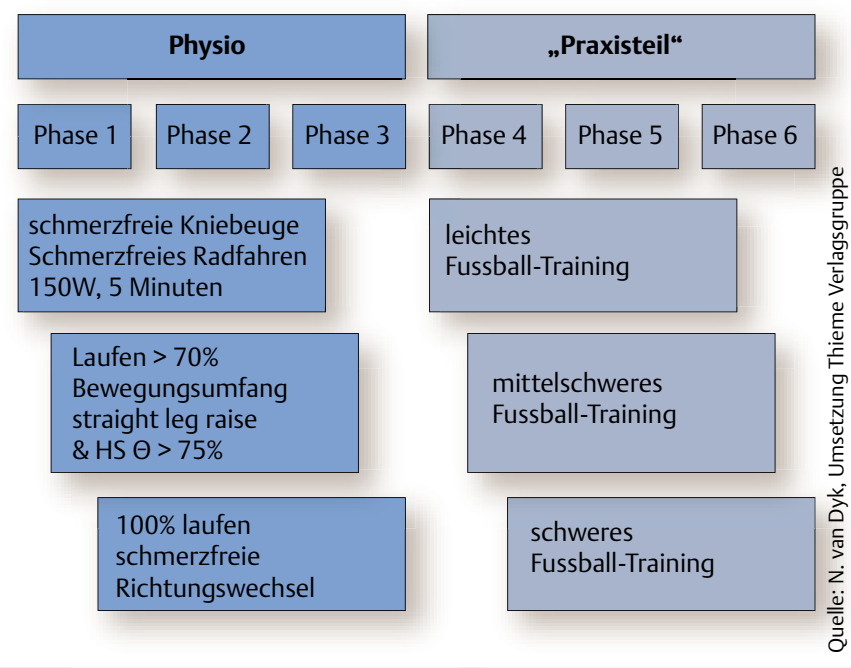

Abb. 1 Kriterienbasierter Verlaufsalgorithmus

\section{TABELLE 1}

\section{Rehabilitationsziele der einzelnen Phasen}

\begin{tabular}{|c|c|c|}
\hline $\begin{array}{l}\text { PHASE 1: Förderung des Heilungs- } \\
\text { prozesses und frühe, optimale Be- } \\
\text { lastung des verletzten Gewebes }\end{array}$ & $\begin{array}{l}\text { PHASE 2-3: Vollständige Wiederherstellung der Mus- } \\
\text { kelfunktion }\end{array}$ & $\begin{array}{l}\text { PHASE 4-6: Integration vollständiger, } \\
\text { sportspezifischer Integration }\end{array}$ \\
\hline
\end{tabular}




\section{Spezifische Übungen für Phase 1}

1. Radfahren auf einem Ergometer

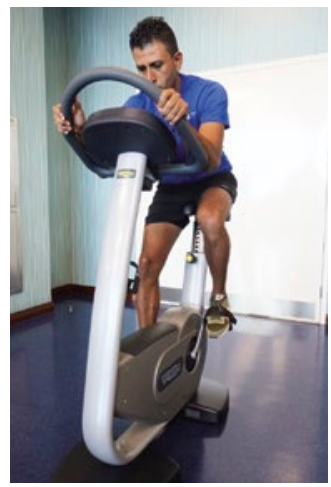

2. beidbeinige Kniebeuge

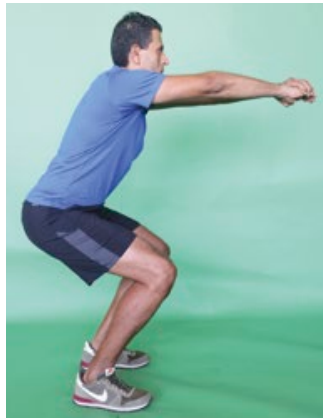

3. einbeinige Kniebeuge

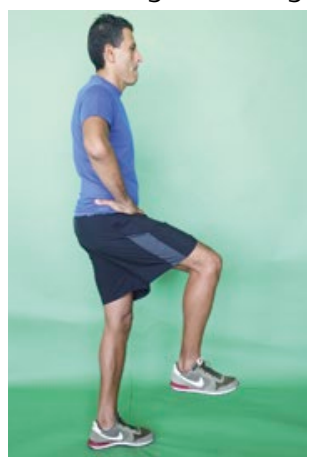

4. Bridging

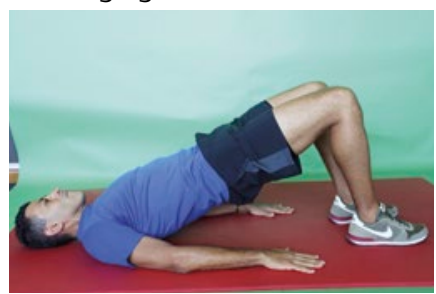

5. isometrische Heel Digs

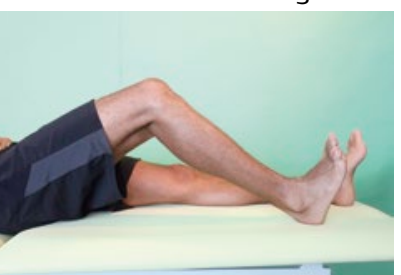

6. Knieflexion gegen manuellen Widerstand

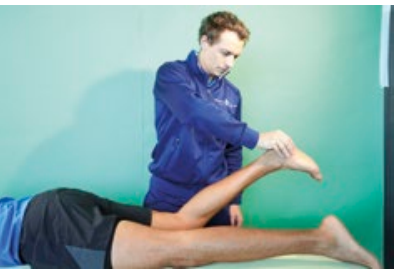

7. aktiver Bewegungsumfang in Knieflexion

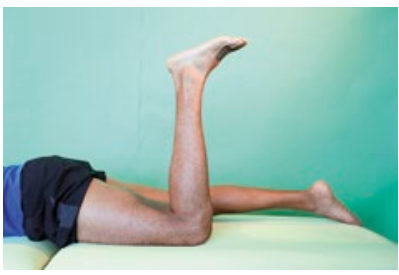

8. einbeiniges Bridging

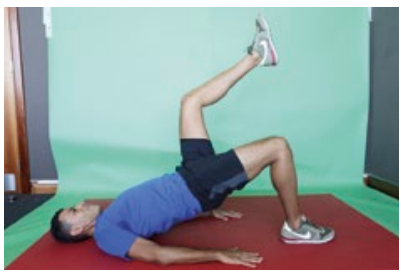

9. Stretching

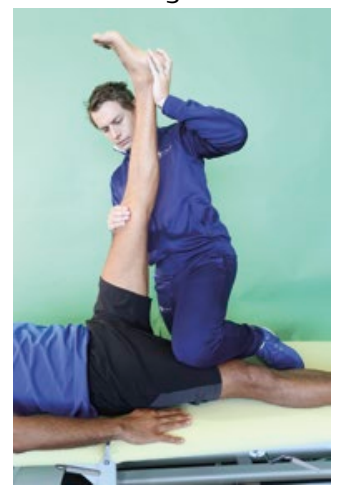

10. Leg Curl (Knieflexion) in Bauchlage

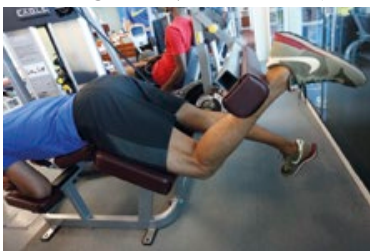

11. Nordic-Hamstring-Übung

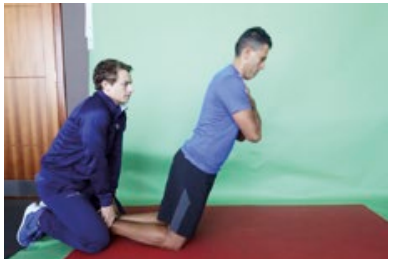

12. Lengthening Exercise - Extender

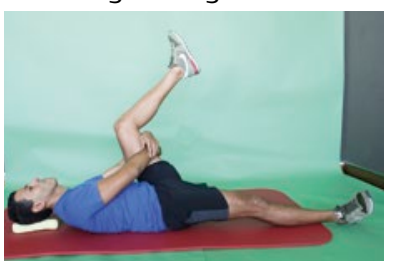

13. Lengthening Exercise - Arabesque/Diver

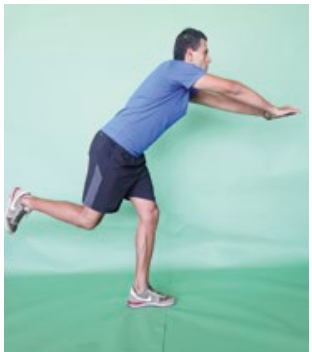

14. Lengthening Exercise - Glider

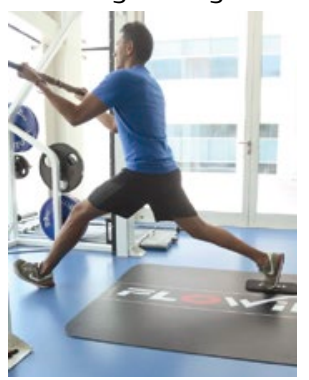




\section{Phase 2}

In Phase 2 wird die Trainingsbelastung schrittweise gesteigert. Die Übungsausführung muss sorgfältig überwacht und gegebenenfalls korrigiert werden.

In dieser Phase beginnt man mit Kraftübungen mit zunehmendem Schwierigkeitsgrad sowie mit dem Protokoll für die Steigerung des Lauftrainings. Typischerweise bedeutet dies einen Übergang zu einbeinigen Übungen, höheren Laufgeschwindigkeiten und Übungen unter Verlängerung der Muskulatur. Falls angemessen können die exzentrischen Lengthening Exercises eingeführt werden [4]. Bei Verschlechterung von Muskelkraft oder Bewegungsumfang respektive bei einer Schmerzzunahme muss die Belastung bei den problematischen Übungen reduziert werden.

Das Monitoring der Reaktion des Sportlers durch tägliche Messungen (Schmerzen, Palpation, Kraft und Flexibilität) hilft dabei, die Reaktion auf die Belastung zu bestimmen und die Frage zu beantworten, ob der Sportler bereit für die nächste Phase ist, auf dem aktuellen Niveau weitertrainieren oder zur vorherigen Phase zurückkehren sollte.

Lauftraining In dieser Phase wird mit dem eigentlichen Lauftraining begonnen. Die wichtigsten Punkte des Protokolls für das Lauftraining sind Umfang, Intensität und die biomechanischen Aspekte des Laufens. Es wird unter Beobachtung ausgeführt, um zu gewährleisten, dass diese Komponenten richtig ein- und umgesetzt und, falls erforderlich, angepasst werden können.

Vor dem Laufen absolviert der Sportler ein angemessenes Aufwärmprogramm. Dieses Programm kann Radfahren auf dem Ergometer, langsames Laufen oder andere Kardioübungen für die untere Extremität beinhalten. Vor allem muss darauf geachtet werden, dass die Sprint-Techniken „A“ und „B“ richtig ausgeführt werden. Bei diesen Übungen liegt der Schwerpunkt auf dem letzten Abschnitt der Schwungphase bzw. der dreifachen Extensionsphase. Sie werden bilateral ausgeführt und bieten die Möglichkeit, Asymmetrien in diesen Bewegungsphasen aufzuspüren und gegebenenfalls zu korrigieren.

Bei der Wiederaufnahme des Lauftrainings muss darauf geachtet werden, dass die Belastung beim Laufen progressiv und vorsichtig erhöht wird. Unserer Erfahrung nach ist es hilfreich, den Sportler selbst zu bitten, den Grad der körperlichen Anstrengung beim Laufen einzuschätzen. Auf diese Weise kann gewährleistet werden, dass die Belastung (Laufgeschwindigkeit) über mehrere Trainingseinheiten auf demselben Niveau bleibt und vorsichtig erhöht werden kann, sobald der Sportler eine bestimmte Geschwindigkeit erreicht hat, ohne dass Probleme auftreten.

Typischerweise wird die Bewertung mithilfe einer Skala von 0 bis $100 \%$ vorgenommen (s. Abb. 2c), wobei $100 \%$ einem Sprint mit maximaler Anstrengung/Geschwindigkeit entspricht und 0\% der langsamsten Geschwindigkeit, in der der Sportler laufen kann. Gelaufen wird auf einer ovalen Bahn, die aus ca. 30 Metern Geraden und ca. 100 Metern Kurven besteht (Abb. 2a). Der Sportler beginnt den „Lauf“ aus dem Gehen (also nicht aus dem Stand) heraus am Anfangspunkt einer Geraden und verlangsamt sein Tempo, wenn er in die Kurve eintritt. Nach jeder Trainingseinheit (4 Runden, 8 „Läufe auf der Geraden“) wird er gebeten einzuschät-

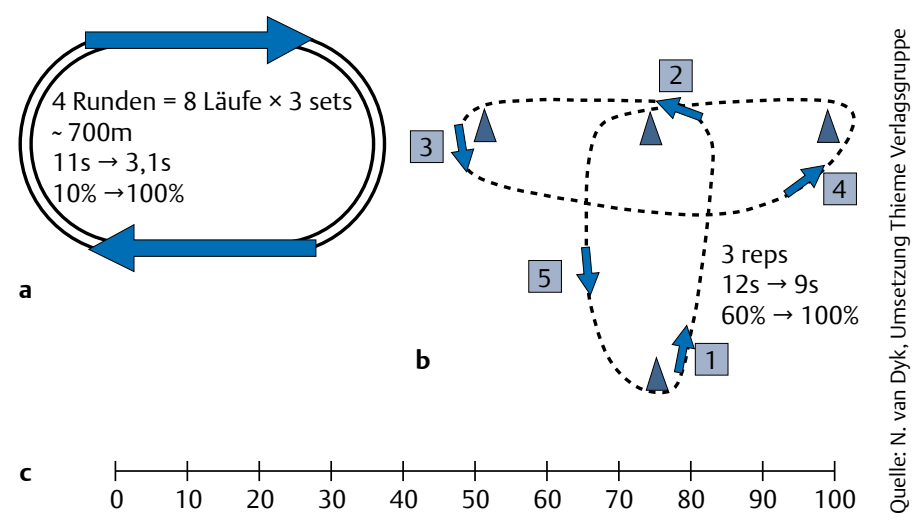

Abb. 2 Lauftraining und Richtungswechsel a) Laufen auf der Bahn, $30 \mathrm{~m}$ Geraden und $100 \mathrm{~m}$ Kurven. 4 Runden $=8$ Sprints (jeweils auf der Geraden), davon 3 Sätze (also Durchgänge à 4 Runden) - dies entspricht ca. 700m. b) Modifizierter T-Drill; Lauf startet bei Nr. 1 und folgt dann den Zahlen der Reihe nach um die Kegel herum. c) \% lineare Skala, auf der der Spieler angeben soll, mit welcher Geschwindigkeit er gelaufen ist.

zen, wie hoch seiner Meinung nach seine Höchstgeschwindigkeit während dieser Trainingseinheit war. Mit einer Stoppuhr wird gemessen, wie lange er für die 30 Meter lange Gerade braucht. In der Praxis entsprechen 0-10\% für gewöhnlich ungefähr 13 bis 15 Sekunden, während ein Höchstgeschwindigkeitssprint (100\%) eine Dauer von nur 2,9 Sekunden haben kann.

Wenn der Spieler eine ganze Trainingseinheit ohne Schmerzzunahme absolvieren kann, wird die Laufgeschwindigkeit um 5 bis $10 \%$ erhöht. Wenn dies zu Problemen führt - vielleicht fühlt sich der Spieler nicht wohl mit der höheren Geschwindigkeit, oder es treten Schwierigkeiten in Bezug auf die Bewegungsmechanik oder -kontrolle auf -, wird die Laufgeschwindigkeit wieder auf den zuletzt erreichten Wert verringert. Wenn beim Laufen Probleme oder Schmerzen auftreten, wird die Übung abgebrochen und auf die nächste Session verschoben.

In unserer Rehabilitation beträgt die Strecke, die ein Spieler pro Session normalerweise zurücklegt, ungefähr 700 Meter (3 Sets zu je 4 Runden oder 8 „Läufen auf der Geraden“). Das entspricht ziemlich genau der Strecke, die ein Profifußballer in einem Spiel durch Sprinten zurücklegt [6].

\section{Kriterien für den Übergang zu Phase 3}

Der Spieler muss in der Lage sein, mit mehr als 70\% der Höchstgeschwindigkeit (laut Selbsteinschätzung) zu laufen. Davon abgesehen muss der Kliniker entscheiden, ob Kraft und Flexibilität ausreichend wiederhergestellt sind, um die nächste Phase in Angriff zu nehmen. Als ausreichend gelten 75\% schmerzfreier Bewegungsumfang und 75\% Muskelkraft im Verhältnis zum kontralateralen Bein.

In Hassans Fall gab es hier keinerlei Probleme. Nach 10 Tagen in Phase 2 (15 Tage insgesamt) konnte er zu Phase 3 übergehen. Die Muskelkraft betrug $80 \%$ im Vergleich zum kontralateralen Bein, der Bewegungsumfang war zu 100\% wiederhergestellt. 


\section{Phase 3}

In Phase 3 werden die Kraftübungen sowie die exzentrischen Lengthening Exercises intensiviert [4]. Hinzu kommen exzentrische Kraftübungen, vor allem die Nordic-Hamstring-Übung [18]. Belastung und Geschwindigkeit der Dehnungsübungen werden erhöht, um den Schwierigkeitsgrad zu steigern. Das Lauftraining wird um Richtungswechsel erweitert, die Laufgeschwindigkeit auf $100 \%$ (laut Selbstbewertung) erhöht.

Das Monitoring des Sportlers durch tägliche Messungen (Schmerzen, Palpation, Kraft und Flexibilität) hilft dabei, die Reaktion auf die Belastung zu bestimmen und die Frage zu beantworten, ob der Sportler bereit für die nächste Phase ist, auf dem aktuellen Niveau weitertrainieren oder zur vorherigen Phase zurückkehren sollte. Falls erforderlich, kann ein „Ruhetag“ eingelegt werden, damit sich der Sportler zwischen zwei Behandlungssitzungen erholen kann.

Die Kraftübungen können nun spezifischer auf die individuellen Bedürfnisse und Fähigkeiten des Sportlers abgestimmt werden. Hassan führte die exzentrischen Lengthening Exercises in dieser Phase auch mit zusätzlichen Gewichten und/oder höherer Geschwindigkeit aus, um ihre Wirksamkeit zu erhöhen.

Lauftraining - Richtungswechsel Das Lauftraining wird intensiviert durch Einbeziehung von Richtungswechseln in Form eines modifizierten T-Drills. Der Spieler wird aufgefordert, aus dem Stand heraus loszulaufen und in einer kontinuierlichen Vorwärtsbewegung alle Kegel zu berühren und dabei die Richtung zu ändern, ohne Seitwärtsschritte zu machen oder rückwärts zu laufen. Der Grundgedanke hinter dieser Übung ist, dass Richtungswechsel in einer kontinuierlichen Vorwärtsbewegung eher dem tatsächlichen Geschehen auf dem Spielfeld entsprechen. In diesem Sinne ist diese Übung eine Vorwegnahme des „Praxisteils“ der Rehabilitation, allerdings mit geringerer Belastung (siehe Abb. 2b).

Auch für Richtungswechsel gilt, dass sie erst dann trainiert werden sollten, wenn der Spieler mindestens 70\% der Höchstgeschwindigkeit problemlos erreicht. Ist dieses Kriterium erfüllt, kann der Spieler mit dem Training von Richtungswechseln bei einer Geschwindigkeit von 70\% beginnen; diese wird dann immer weiter erhöht. Gemessen wird die Zeit, die der Spieler für einen vollständigen Durchgang des T-Drills benötigt.

\section{Kriterien für den Übergang zu den Phasen 4-6}

Wenn der Spieler mit $100 \%$ der Höchstgeschwindigkeit (laut Selbsteinschätzung) laufen und den modifizierten T-Drill mit maximaler Geschwindigkeit (laut Selbsteinschätzung) absolvieren kann, ist er bereit für die Phasen 4-6.

In unserem Fallbericht erfüllte Hassan 19 Tage nach der Verletzung die Kriterien für den Übergang zu den sportspezifischen Phasen des Protokolls.

\section{Phase 4-6: sportspezifische Rehabilitation}

Nach Abschluss der dritten Rehabilitationsphase beginnt der Spieler mit den Trainingssessions auf dem Spielfeld. Dieses umfasst drei 30- bis 45-minütige sportspezifische Trainingseinheiten. Trainiert werden Laufen, Sprinten, Richtungswechsel und spezifische Spieltechniken wie Passen, Schießen und Störmanöver.

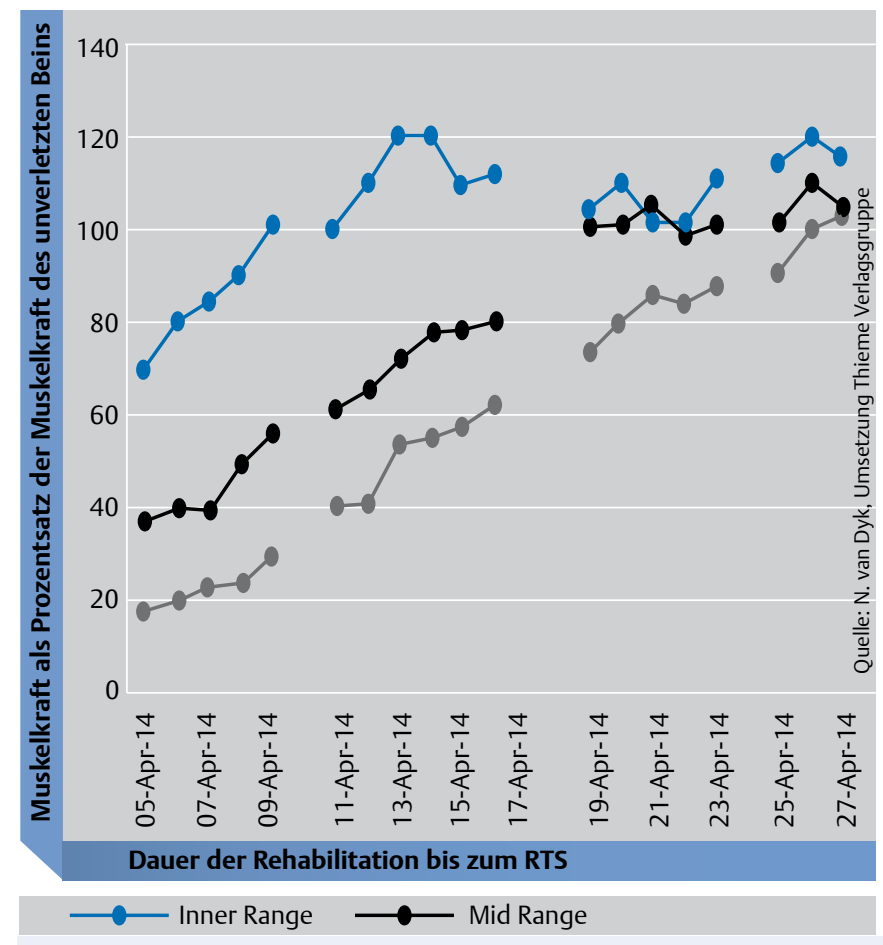

Abb. 3 Entwicklung der Muskelkraft bis zum RTS in diesem Fallbericht. Die Y-Achse stellt die Muskelkraft als Prozentsatz der Muskelkraft des unverletzten Beins dar, die X-Achse die Dauer der Rehabilitation bis zum RTS vom ersten Tag bis zum letzten Tag (Entlassung).

Wenn der Spieler die Kriterien nicht erfüllt oder Schmerzen oder andere Probleme auftreten, wird das Programm im Hinblick auf die Belastung angepasst. Wenn die Symptome nicht verschwinden, wird die vorherige Phase des Protokolls wiederholt.

Nach Beendigung der sportspezifischen Rehabilitationsphasen (Phasen 4-6) und einem abschließenden Assessment (klinische Untersuchungen) wird der Spieler aus der Rehabilitation entlassen.

\section{Return to Sports (RTS) - Assessment und Empfehlung}

Nach Beendigung der Rehabilitation werden die üblichen Standardtests für die Entlassung durchgeführt, die als Grundlage für die RTS-Entscheidung dienen. Die Messungen der Muskelkraft und der Flexibilität werden wiederholt, ebenso die funktionellen Bewegungstests des täglichen Assessment-Formulars (Abb. 4).

Zusätzlich wird ein isokinetisches Assessment durchgeführt, der Askling H-Test [5], und der Patient wird gebeten, selbst zu bewerten, ob bzw. in welchem Maß er sich bereit fühlt, seinen Sport wieder auszuüben. Diese Information fließt in die RTS-Entscheidung mit ein, an der unserer Meinung nach der Spieler, der behandelnde Physiotherapeut und der Mannschaftsarzt bzw. der mit der Behandlung betraute Sportarzt beteiligt sein sollten.

\section{Isokinetisches Assessment}

Die Muskelkraft bei Knieflexion und -extension werden mithilfe eines isokinetischen Dynamometers gemessen (Biodex Multi- 


\begin{tabular}{|c|c|c|c|}
\hline \multirow[b]{3}{*}{ durchschnittliche Schmerzen heute } & \multicolumn{3}{|c|}{$\begin{array}{l}\text { / / 201.... wievielter Tag? } \\
\text { Zeichen; } \\
\text { Unterschrift des Klinikers }\end{array}$} \\
\hline & \multicolumn{2}{|c|}{ verletzt } & \multirow{2}{*}{ 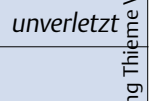 } \\
\hline & VAS & $/ 10$ & \\
\hline Gehen & No & $\mathrm{P} \quad \mathrm{NA}$ & \\
\hline Joggen, Laufen & No & $\mathrm{P} \quad \mathrm{NA}$ & E. \\
\hline Kniebeuge auf beiden Beinen x 3 & No & NA & \\
\hline Kniebeuge auf einem Bein x 3 & No & $\mathrm{P} \quad \mathrm{NA}$ & $i$ \\
\hline Rumpfflexion & No & $\mathrm{P} \quad \mathrm{NA}$ & \\
\hline gesamte Palpation Länge & \multicolumn{2}{|r|}{$\mathrm{cm} P$} & \\
\hline mittlerer Bereich der Bewegungsbahn & \multicolumn{2}{|r|}{$\mathrm{kg} \boldsymbol{P} \mid$ no } & $\mathrm{kg}$ \\
\hline äußerer Bereich & \multicolumn{2}{|r|}{$\mathrm{kg} \boldsymbol{P} \mid$ no } & $\mathrm{kg}$ \\
\hline SLR & \multicolumn{2}{|r|}{${ }^{\circ} \boldsymbol{P} \mid$ no } & $\circ$ \\
\hline MHFAKE & \multicolumn{2}{|r|}{${ }^{\circ} \boldsymbol{P} \mid$ no } & $\circ$ \\
\hline Brücke mit angewinkelten Beinen x 3 & No & NA & \\
\hline Brücke mit gestreckten Beinen x 3 & No & $P \quad N A$ & \\
\hline Bemerkungen: & & & \\
\hline
\end{tabular}

Abb. 4 Aspetar-Formular für das tägliche Assessment zur Überwachung des Rehabilitationsverlaufs und zur Unterstützung des Clinical Reasoning

Joint System 3). Die Testreihe umfasst drei unterschiedliche Modi und Geschwindigkeiten: 1. konzentrische Knieflexion und -extension bei $60 \% \mathrm{~s}(5 \mathrm{Mal}) ; 2$. konzentrische Knieflexion und -extension bei $300 \% \mathrm{~s}(10 \mathrm{Mal}) ; 3$. exzentrische Knieextension bei $60 \% \mathrm{~s}(5$ Mal). Aufgezeichnet wird jeweils der höchste bei sämtlichen Wiederholungen erreichte Drehmomentmaximalwert.

Askling H-Test Der Patient liegt auf dem Rücken, das kontralaterale Bein und der Oberkörper werden mit einem Gurt fixiert. Ein Kniebrace am zu testenden Bein gewährleistet die vollständige Extension des Knies $\left(0^{\circ}\right)$. Die Testreihe beginnt mit dem passiven Flexibilitätstest, bei dem der Kliniker das Bein langsam anhebt, bis die Hüfte maximal flektiert ist. Der Endpunkt der Bewegungsbahn ist erreicht, wenn der Patient eine starke, aber ertragbare Dehnung der Hamstring-Muskeln verspürt.

Beim anschließenden aktiven Flexibilitätstest wird zuerst ein Probedurchlauf mit submaximaler Intensität durchgeführt. Anschließend wird der Test drei Mal „mit maximaler Intensität" wiederholt. Der Test besteht darin, das gestreckte Bein so schnell wie möglich und so hoch wie möglich anzuheben, ohne dabei ein Verletzungsrisiko einzugehen. Anschließend wird der Patient gebeten, mithilfe einer visuell-analogen Skala von 0 bis $100 \mathrm{zu}$ beschreiben, wie sicher er sich beim Ausführen der Beinbewegung gefühlt hat und ob dabei Schmerzen aufgetreten sind [5].
Hassan wurde 23 Tage nach seiner Verletzung aus der Rehabilitation entlassen, die insgesamt 20 Tage gedauert hatte. Zusammen mit dem Mannschaftsphysiotherapeuten überwachten wir sein RTS. Sieben Tage nach seiner Entlassung nahm er erstmals wieder an einem Spiel teil. Er absolvierte den Rest der Saison ohne weitere Verletzungen, und auch die rehabilitierte Hamstring-Verletzung hatte keine weiteren Auswirkungen auf seine Leistung.

\begin{tabular}{l} 
:: Wir empfehlen Spielern, Trainern und Betreuern, \\
\hline den RTS schrittweise zu bewerkstelligen: \\
- 1 x 50 \%ige Trainingssession \\
- 2 x vollständige Trainingssession \\
- eingeschränkter Einsatz beim ersten Spiel nach der \\
Rehabilitation ( $50 \%$ oder 30 Minuten) \\
- uneingeschränkter Einsatz
\end{tabular}

\section{Tägliche physische Assessments zur Überwachung des Rehabilitationsverlaufs}

Unsere täglichen physischen Assessments beinhalteten subjektive Schmerzempfindung (visuell-analoge Skala), Schmerzen bei Palpation/Druckempfindlichkeit, funktionelle Bewegung, Muskelkraft und Bewegungsumfang. Diese Messungen bildeten die Grundlage für das Clinical Reasoning, bei dem von Tag zu Tag darüber entschieden wurde, wie die Behandlung des Spielers fortgesetzt oder verändert werden sollte (siehe Abb. 4-13).

Der Spieler gibt jeden Tag darüber Auskunft, ob und wie starke Schmerzen er hat, und das Ausmaß des Schmerzgebietes bei Palpation wird gemessen. Um die Funktion zu bewerten, führt der Spieler eine standardisierte Kniebeuge, eine einbeinige Kniebeuge sowie eine Rumpfflexion aus.

Flexibilität Es wird sowohl der aktive als auch der passive Bewegungsumfang gemessen. Beim passiven Straight-Leg-Raise-Test (Hüftflexion) liegt der Spieler auf dem Rücken und der Kliniker fixiert das kontralaterale Bein (Abb. 11). Ein Hand-Theta-WinkelInklinometer wird auf halber Höhe auf der vorderen Schienbeinkante platziert, während der Kliniker das Bein des Spielers anhebt, wobei er mit dem Arm für eine vollständige Knieextension sorgt. Der Winkel wird entweder gemessen, wenn der Endpunkt des Straight Leg Raise (maximale Hüftflexion) erreicht ist oder wenn Schmerzen auftreten.

Abb. 12 zeigt eine Variante des aktiven Knieextensionstests, die in maximaler Hüftflexion ausgeführt und als „Maximal Hip Flexion Active Knee Extension Test“ (MHFAKE) bezeichnet wird [13]. Dabei werden die Arme hinter dem Oberschenkel verschränkt, um die Hüfte in maximaler Flexion zu halten, während der Spieler das Knie aktiv extendiert, bis die maximal ertragbare Dehnung des Hamstring-Muskels erreicht ist. Das kontralaterale Bein wird dabei vom Kliniker fixiert. Der absolute Knieextensionswinkel wird am Endpunkt der maximal ertragbaren Dehnung gemessen, indem ein Hand-Inklinometer auf halber Höhe auf der vorderen Schienbeinkante platziert wird. 

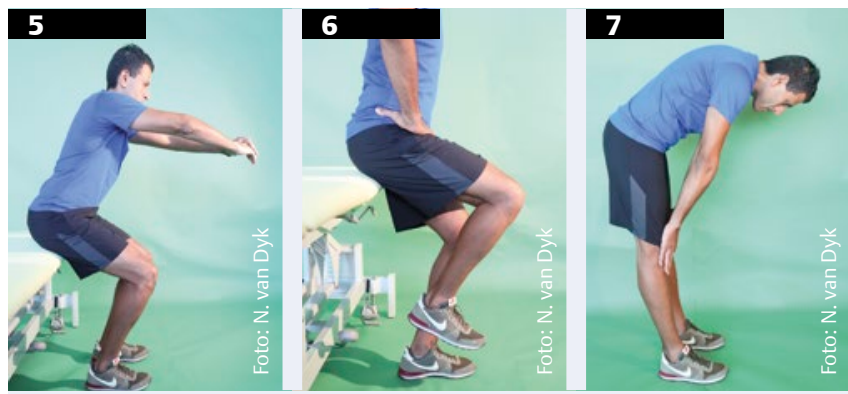

Abb. 5 Kniebeuge auf $90^{\circ}$ : Der Patient steht vor einer ca. kniehohen Bank und senkt den Oberkörper, indem er die Knie beugt, bis er die Bank berührt. Die Knie sollen sich dabei genau über den Zehen befinden.

Abb. 6 Einbeinige Kniebeuge auf $45^{\circ}$ : Der Patient steht vor einer bis zur Mitte der Oberschenkel hohen Bank und senkt den Oberkörper, indem er die Knie beugt, bis er die Bank berührt. Die Knie sollen sich dabei genau über den Zehen befinden.

Abb. 7 Rumpfflexion. Der Spieler steht aufrecht und beugt den Oberkörper vor, indem er die Hände an den Oberschenkeln herabgleiten lässt. Die Bewegung wird gestoppt, sobald Schmerzen oder andere Probleme auftreten. Das Ausmaß der Rumpfflexion wird aufgezeichnet, das heißt, bis wohin die Finger reichen: Schienbeinmitte, Sprunggelenk, Zehen oder Boden.
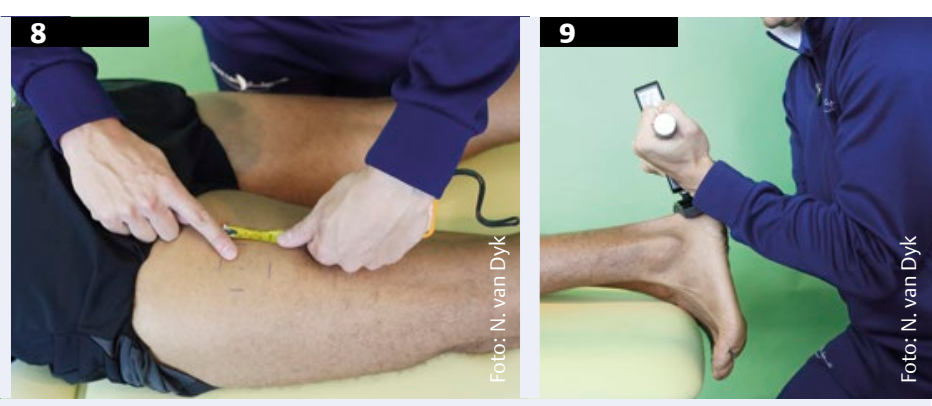

Abb. 8 Palpation: Länge und Breite des schmerzenden Bereichs werden nach den Angaben des Spielers während der Palpation vermessen.

Abb. 9 Messung der Muskelkraft im mittleren Bereich der Muskellänge (es geht um das Ausmaß der Überlappung der Filamente). a) Ausgangsposition: Der Spieler liegt auf dem Bauch, während der Kliniker den Unterschenkel anhebt, bis der Abstand zwischen Fuß und Behandlungsbank ca. $30 \mathrm{~cm}$ beträgt. b) Der Kliniker hält ein HandDynamometer gegen die Ferse und drückt damit den Fuß nach unten, mit einem exzentrischen Bremstest nach drei Sekunden isometrischen Haltens.
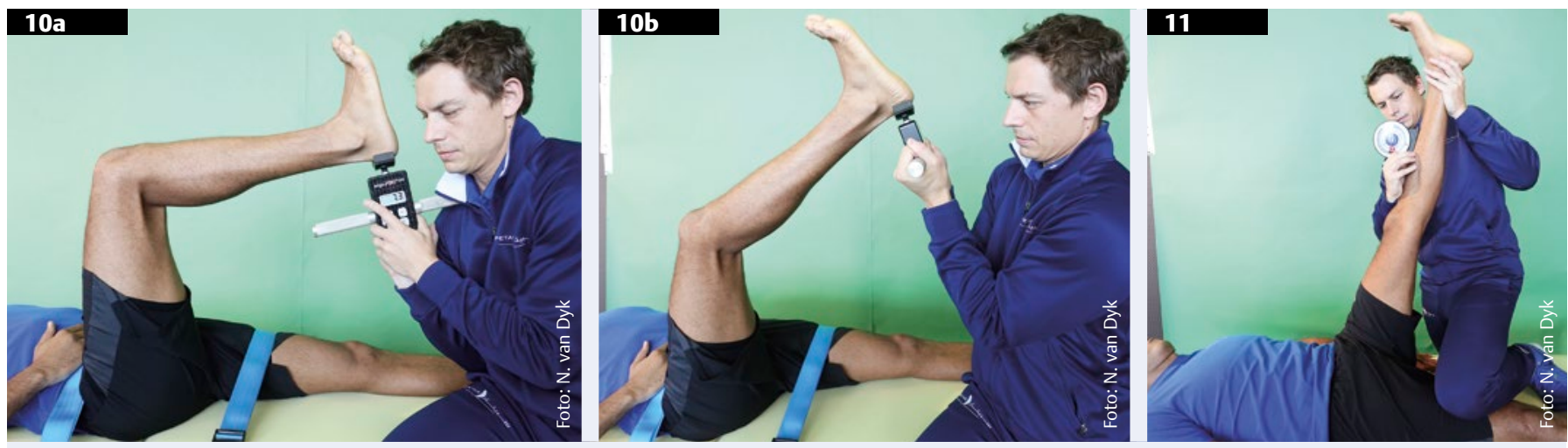

Abb. 10 Messung der Muskelkraft im äußeren Bereich der Muskellänge - heißt in verlängertem Zustand des Muskels (weniger Überlappung der Filamente). a) Ausgangsposition: Der Spieler liegt auf dem Rücken, die Hüfte und das kontralaterale Bein sind mit einem Gurt fixiert. Der Kliniker positioniert das Bein in $90^{\circ}$ Hüft- und Knieflexion und misst mit einem tragbaren Dynamometer den Widerstand, den der Untersuchte gerade noch halten kann - drei Sekunden isometrisches Halten. b) Der Untersucher hält das tragbare Dynamometer gegen die Ferse und drückt damit den Fuß nach oben - exzentrischer Bremstest nach drei Sekunden isometrischen Haltens. bei er darauf achtet, dass das Knie vollständig extendiert bleibt. Der
Abb. 11 Passiver Straight-Leg-Raise-Test. Der Spieler liegt auf dem Rücken, das unverletzte Bein wird in bequemer Position ausgestreckt. Der Kliniker stabilisiert das unverletzte Bein, während er das verletzte Bein bis zur maximal ertragbaren Hüftflexion anhebt, woSpieler gibt an, ab welchem Winkel Schmerzen in den Hamstrings auftreten bzw. wann die maximal ertragbare Dehnung erreicht ist.

namometer (TD) mit beiden Händen in einer bequemen Position gegen die Ferse, wobei der Winkel des TD berücksichtigt wird. Der Test erfordert, dass der Spieler drei Sekunden lang eine maximale willkürliche isometrische Kontraktion gegen das TD ausführt.

Um die Muskelkraft bei verlängertem Muskel zu messen, nimmt der Spieler die Rückenlage ein und das Becken wird mit
Muskelkraft Die Muskelkraft in mittlerer und verlängerter Muskellänge (bezüglich der Überlappung) wird jeden Tag gemessen. Um die Muskelkraft im mittleren Teil der Muskellänge zu messen, nimmt der Spieler die Bauchlage ein und der Kliniker flektiert das Knie, bis der Abstand zwischen Fuß und Behandlungstisch etwa $30 \mathrm{~cm}$ beträgt (Abb. 9). Der Kliniker steht hinter dem Spieler und hält ein Hand-Dy- 

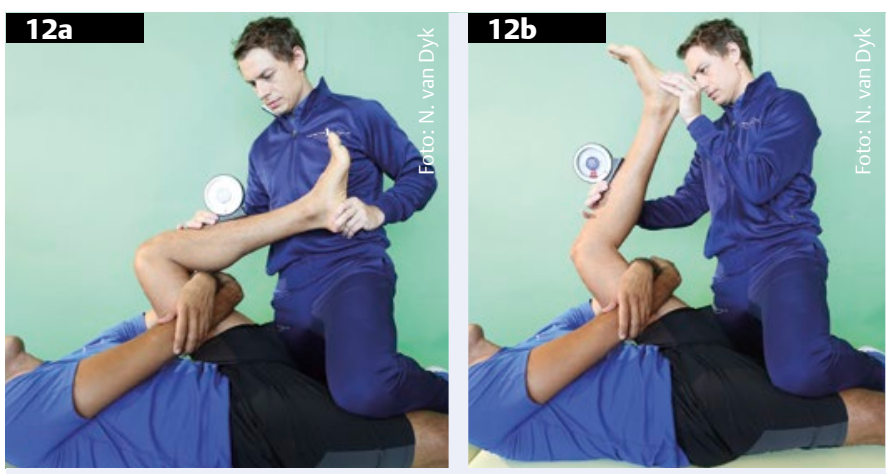

Abb. 12 Maximal Hip Flexion Active Knee Extension (MHFAKE). a) Ausgangsposition: Der Spieler liegt auf dem Rücken und zieht das verletzte Bein an den Oberkörper heran, bis die maximale Hüftflexion erreicht ist, und bleibt dann in dieser Position. Der Kliniker fixiert dabei das andere Bein. b) Dann extendiert der Spieler das Knie so weit wie möglich. Der erreichte Bewegungsumfang wird mit einem Inklinometer gemessen.
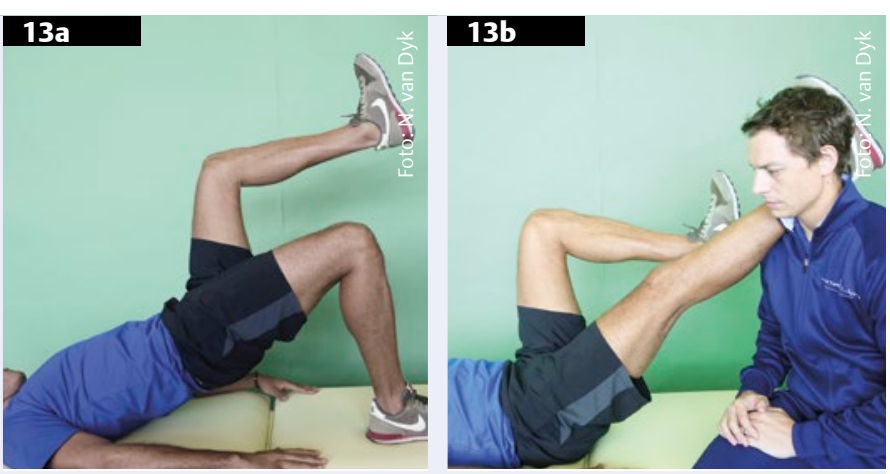

Abb. 13 Endposition der Brücke mit einem Bein. Der Spieler liegt auf dem Rücken und hebt die Hüften in maximale Flexion, während er a) das kontralaterale Bein mit angewinkeltem Knie angehoben hält oder b) das Bein gestreckt auf der Bank oder der Schulter des Klinikers ablegt. einem Gurt fixiert, der in einer Linie mit der Spina iliaca anterior superior liegt (Abb. 10). Der Kliniker flektiert das Knie des zu testenden Beins auf $90^{\circ}$, während das kontralaterale Bein flach auf dem Behandlungstisch liegen bleibt. Der Kliniker steht seitlich neben dem Behandlungstisch und hält ein TD mit beiden Händen und vertikal positioniert gegen die Ferse des Spielers. Der Test erfordert, dass der Spieler drei Sekunden lang eine maximale willkürliche isometrische Kontraktion gegen das TD ausführt. Dazu erhält er standardisierte Instruktionen [24].

In klinischer Hinsicht halten wir die Messung der Muskelkraft in verlängertem Zustand des Muskels für die Messung, die am besten geeignet ist, um als Grundlage für eine Entscheidung über den RTS zu dienen. In Abb. 3 sind die Verbesserungen aller Kraftmessungen in diesem Fallbeispiel dargestellt. Die Muskelkraft bei verlängerter Muskulatur war nach 23 Tagen vollständig wiederhergestellt (gemessen an der Muskelkraft des anderen Beins), was ziemlich genau der Dauer der Rehabilitation bis zum Tag der Entlassung entspricht. Die Wiederherstellung der Muskelkraft bei mittlerer Muskelüberlappung verlief ebenfalls gut, aber die Muskelkraft in diesem Bereich normalisierte sich sehr viel schneller, d.h. innerhalb weniger Tage. Diese Messung war also wenig hilfreich, um eine Aussage über die Fortschritte in der Rehabilitation dieses Sportlers zu erhalten. Wir hatten das Glück, unseren Patienten fünf Mal pro Woche zu sehen, aber wenn das nicht möglich ist, kann die Messung der Muskelkraft unter Verlängerung der Muskulatur verwendet werden, um den Verlauf der Rehabilitation zu verfolgen.

\section{Klinische Prädiktoren für den Return to Sport (RTS)}

Es gibt immer noch keinen Konsens darüber, welche klinischen Messungen am genauesten darüber Aufschluss geben, wie viel Zeit ein verletzter Sportler braucht, bis er seinen Sport wieder ausüben kann. In einer aktuellen Studie berichten Jacobsen und
Kollegen, dass die täglichen physiotherapeutischen Messungen (wie wir in diesem Fallbericht gezeigt haben) dem Kliniker dabei helfen können, die wahrscheinliche Dauer der Rehabilitation einzuschätzen [13].

Mithilfe einer Regressionsanalyse entwickelten die Autoren eine gewichtete Gleichung mit einer Kombination von Eigenschaften, die in den klinischen Untersuchungen am 1. und 7. Tag leicht gemessen werden können. Interessanterweise trugen die MRI-Befunde nicht dazu bei, die Aussagekraft der Gleichung in Bezug auf die Vorhersage des RTS-Zeitpunkts zu verbessern [13]. Auch andere Studien kamen zu dem Ergebnis, dass MRI-Untersuchungen zusätzlich zur Krankengeschichte und den klinischen Untersuchungen kaum einen Mehrgewinn liefern [23] und keine Aussage bezüglich des Zeitpunktes des RTS machen [16]. Über die Aussagekraft von MRI-Untersuchungen wird jedoch nach wie vor gestritten, und es gibt auch Studien, die zu anderen Schlussfolgerungen kommen $[9,10]$. Fest steht nur, dass Vorsicht geboten ist, wenn MRI-Befunde im Hinblick auf den RTS interpretiert werden sollen.

Eine Kombination von klinischen Befunden mit $97 \%$ der erklärten Varianz ( \pm 5 Tage) bei der Nachfolgeuntersuchung am 7. Tag könnte eine gewisse Vorhersagekraft bezüglich des Zeitpunkts des RTS haben [13].

\section{Die wichtigen subjektiven, mit dem Zeitpunkt des RTS}

\section{assoziierten Punkte waren:}

die maximalen Schmerzen (VAS 1-10), die zum

Zeitpunkt der Verletzung protokolliert wurden

- ein verzögerter Beginn der Physiotherapie

- die Zeit, die der Sportler brauchte, um wieder

schmerzfrei gehen zu können 


\section{:: Die physischen Befunde, die als hilfreich eingestuft} wurden, waren Variablen, die mit den Messungen der Muskelkraft zusammenhingen:

- Veränderung der Schmerzen beim Test der Muskelkraft im mittleren Bereich der Muskellänge während der ersten Woche

- Schmerzen beim Test der Muskelkraft im verlängerten Bereich und beim einbeinigen Bridging am 7. Tag

- Muskelkraft unter Muskelverlängerung am 7. Tag, ausgedrückt als Prozentsatz der Muskelkraft des unverletzten Beins

- maximale isokinetische Kraft der Knieflexion des unverletzten Beins

Sorgfältig durchgeführte Messungen können dem Kliniker Anhaltspunkte dafür liefern, wie lange die Rehabilitation bis zum RTS eines Fußballspielers wahrscheinlich dauern wird.

\section{Diskussion/Zusammenfassung}

In diesem Fallbericht haben wir ein kriterienbasiertes Rehabilitationsprotokoll für einen Fußballspieler mit einer typischen akuten, beim Laufen erlittenen Hamstring-Verletzung vorgestellt. Wir sind davon überzeugt, dass der Wechsel von einem zeitbasierten Ansatz zu einem auf Kriterien basierenden Verlaufsprotokoll die Erfolgsaussichten einer Rehabilitation enorm verbessern. Dieser neue Ansatz erlaubt es dem Kliniker, sowohl objektive Messungen als auch subjektive Bewertungen in das Clinical Reasoning zu integrieren.

In der Fachliteratur werden verschiedene Rehabilitationsprotokolle beschrieben [17]. Die Verschiedenheit all dieser Ansätze hat dazu beigetragen, unser Verständnis und unser Wissen darüber, was ein gutes Rehabilitationsprotokoll ausmachen sollte, zu verbessern. Die Schwierigkeit des vielfältigen Angebots an Interventionen besteht darin zu erkennen, wie und wann eine bestimmte Intervention einem verletzten Spieler helfen kann.

Ein wichtiger Aspekt des in diesem Fallbericht vorgestellten Protokolls ist, dass das Lauftraining darin großen Raum einnimmt. Unterschiedliche Arten von Verletzungen erfordern unterschiedliche Behandlungsansätze, und dieses Protokoll ist auf HamstringVerletzungen ausgerichtet, die sich beim Laufen mit hoher Geschwindigkeit ereignen können, das heißt, sie sind typisch für Sportarten, bei denen Laufen eine der Hauptkomponenten ist.

Besonders wichtig für den Erfolg der Rehabilitation ist die multidisziplinäre Zusammenarbeit. Modelle, in denen Entscheidungen gemeinsam getroffen werden können - vor allem im Hinblick auf den RTS -, werden in der Fachliteratur gut beschrieben und haben sich unserer Erfahrung nach bewährt [1, 2, 8]. Nicht nur bei der Entscheidung über den RTS, sondern während des gesamten Rehabilitationsprozesses sind Kommunikation und Absprachen zwischen allen Beteiligten - Ärzte, Therapeuten, Betreuer und natürlich auch der Spieler selbst - erforderlich, um eine Rehabilitation erfolgreich durchzuführen und ein befriedigendes Ergebnis zu erzielen.

Wir haben in diesem Fallbericht eine Kombination aus klinischer Erfahrung und evidenzbasierter Praxis beschrieben, wobei Kriterien für den Behandlungsverlauf und tägliche objektive Messungen die Grundlage des Rehabilitationsprotokolls bildeten. Auf diese Weise konnten die gut dokumentierten Interventionen effektiv und auf angemessene Weise eingesetzt werden. Eine wichtige Rolle bei diesem auf Kriterien basierenden Ansatz spielten die Ergebnisse der physischen Messungen, auf deren Grundlage über den weiteren Verlauf der Rehabilitation entschieden wurde. Diese Vorgehensweise gewährleistet eine methodologische Konsistenz und unterstützt das Clinical Reasoning des Klinikers. Auf diese Weise kann ein standardisiertes Protokoll auf die individuellen Bedürfnisse der Patienten zugeschnitten werden. Dem Kliniker steht eine Vielzahl von Interventionen zur Verfügung, aber um sie auch sinnvoll und zielgerichtet einsetzen zu können, empfehlen wir das in diesem Artikel beschriebene Modell, das Kriterien für den Übergang von einer Phase zur nächsten enthält und für eine effektive und zuverlässige Rehabilitation sorgt.

- Das vollständige Literaturverzeichnis finden Sie unter: www.thieme-connect.de/products/sportphysio

AUTOREN

Nicol van Dyk arbeitet zurzeit als Physiotherapeut in der Abteilung für Rehabilitation am Aspetar Sports Medicine and Orthopaedic Hospital in Doha, Katar. Aus seiner aktuellen Tätigkeit im muskuloskeletalen Vorsaison-Screening von Sportlern entwickelte sich ein besonderes Interesse an Verletzungen der unteren Extremität. Zurzeit untersucht er, welche Risikofaktoren für Profifußballer bestehen, eine Hamstring-Verletzung zu erleiden.

E-Mail: nicol.vandyk@aspetar.com

Arnlaug Wangensteen ist eine norwegische Physiotherapeutin, die kurz vor dem Abschluss ihres PhD-Projektes steht: Diagnosis and prognosis of acute hamstring injuries among male athletes. Die postgraduierte Forscherin arbeitet im Aspetar Sports Medicine and Orthopaedic Hospital in Doha, Katar und im Department of Sports Medicine, Oslo Sports Trauma Research Center, Norwegian School of Sport Sciences.

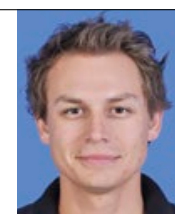

Rod Whiteley ist stellvertretender Leiter der Abteilung für Rehabilitation, Aspetar Sports Medicine Hospital.
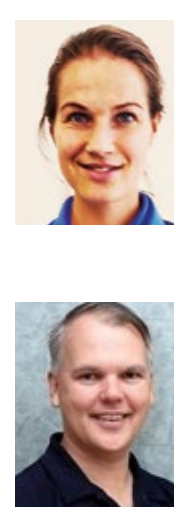

BIBLIOGRAFIE

DOI 10.1055/s-0042-120898

Sportphysio 2017; 5: 22-30

(C) Georg Thieme Verlag KG

Stuttgart · New York · ISSN 2196-5951 